med. Ges. Bd. 31, 1897. - 7. D i M a t t e i, Giornale dell'Acad. di Med. di Torino, 1885. (Zit. nach Oerte l.) - 8. D ü r ck, Studien über die Ätiologie und Histologie der Pneumonie im Kindesalter und der Pneumonie im allgemeinen. D. Arch. f. klin. Med. Bd. 58. - 9. Ell e r m a n n , Störungen der Regeneration von Nierenepithelien. Virch. Arch. Bd. 171, 1903. - 10. $\mathrm{F}$ i s c he r, Über Nierenveränderungen bei Tuberkulösen. Zieglers Beitr. Bd. 47, 1910. 11. Fo à, Ủber Niereninfarkte. Zieglers Beitr. Bd. 45, 1909. - 12. F ri e dlän de r, Virch. Arch. Bd. 68. - 13. He cht, Die Riesenzellenpneumonie im Kindesalter. Zieglers Beitr. Bd. 48, 1910. - 14. He in e k e, Veränderungen der menschl. Niere nach Sublimatvergiftung. 7ieglers Beitr. Bd. 45, 1909. - 15. H e r x h e i m e r, Über die sog. hyaline Degeneration der Glomeruli der Niere. Zieglers Beitr. Bd. 45, 1909. - 16. H o n l, Wiener klin. Rundschau 1897. - 17. Kro ma ye r, Virch. Arch. Bd. 117. - 18. Kr ü ckmann, Virch. Aréh. Bd. 118, Suppl. - 19. L äh $\mathbf{r}$, Über den Untergang des Staphylococcus pyog. aur. in den durch ihn hervorgerufenen Entziundungsprozessen der Lunge. Inaug.-Diss. Bonn 1887. - 20. L o n i c er, Úber Riesenzellenbildung in der Leber bei Lues congenita. Zieglers Beitr. Bd. 45, 1909. - 21. M a r c a n t o n i o, Giornale internazionale delle scienze med. vol. 23, 1901. (Zit. nach $\mathrm{R}$ össle.) - 22. M e t s chnik of f, İber die phagozytäre Rolle der Tuberkelriesenzellen. Virch. Arch. Bd. 113, 1888. - 23. $\mathrm{N}$ a u w $\mathrm{r} \mathrm{ck}$ und $\mathrm{H} \mathrm{n} \mathrm{s} \mathrm{ch} \mathrm{mied,} \mathrm{Über} \mathrm{das}$ multilokuläre Adenozystom der Nieren. Zieglers Beitr. Bd. 12, 1893. - 24. Oe r te l, On epithelial proliferation and the formation of epithelial giant cells in nephritis. Publications of the Russel Sage Institute of Pathology Nr. 1. - 25. $0 \mathrm{p} \mathrm{p} \mathrm{e} \mathrm{n} \mathrm{h}$ e i m e r, Riesenleberzellen bei angeborener Syphilis. Virch. Arch. Bd. 182, 1905. - 26. P e tr o n e, La rigenerazione del fegato e del rene. Morgagni, 11/12, 1881. -- 27. Pels Le us de n, Zur pathologisehen Anatomie der Puerperaleklampsie. Virch. Arch. 142, 1895. - 28. P i s e n ti, Arch. ital. de biol. vol. 6, 1884. (Zit. nach O e r te l.) -- 29. P o d w y s o z k i, Experimentelle Untersuchungen über Regeneration der Drüsengewebe. II. Teil. Regeneration der Nierenepithelien. Zieglers Beitr. Bd. 2, 1888. - 30. D e r s e l b e, Zur Frage über die formativen Reize. Riesenzellengranulome durch Kieselgur hervorgerufen. Zieglers Beitr. Bd. 47, 1910. - 31. P r u tz, Úber das anatomische Verhalten der Nieren bei der puerperalen Eklampsie. Ztschr. f. Geb. u. Gyn. Bd. 22, 1892. - 32. $\mathrm{R}$ ös s le, Störungen der Regeneration von Nierenepithelien. Virch. Arch. Bd. 170, 1902. - 33. Ders el b e, Epitheliale Riesenzellen der Leber bei Tuberkulose. Verhdl. d. D. Path. Ges., 11. Tagung, 1907. - 34. S t e in d l, Riesenzellen im Harnsediment bei Urogenitaltuberkulose. Wiener klin. Wschr. Bd. 23, 1910, S. 1752 u. 1852. - 35. W a ka b a y a s h i, Virch. Arch. Bd. 204, 1911. -- 36. W e i g e r t, Sammlung klin. Vorträge von Volkmann, 1878/79, Nr. 162/163. - 37. D e r s e l b e, Zur Theorie der tuberkulösen Riesenzellen. D. med. Wschr. Bd. 11, 1885. - 38. W y g o d z i n s k i, Inaug.-Diss. Berlin 1898.

\title{
XIX.
}

\section{Eine seltene anatomische Form von Mitralstenose.}

(Aus dem Pathologischen Institut in StraBburg.)

Von

M ax Bus c h.

(Hierzu 2 Textfiguren.)

Am 2. Dezember $1907 \mathrm{kam}$ im hiesigen Pathologischen Institute die Leiche der am 30. November 1907 in der medizinischen Universitätsklinik verstorbenen 65 Jahre alten Näherin Julie S. zur Autopsie.

Der Tod war unter den Erscheinungen einer Herzinsuffizienz erfolgt.

Aus der Krankengeschichte, die Herr Privatdozent Dr. v. Tabora, Oberarzt der Klinik, in dankenswerter Weise zur Einsichtnahme bereitwillig zur Verfügung stellte, gebe ich folgende Daten wieder. 
A n a m nese: Die Mutter der Patientin starb an „Engigkeit", der Vater an einer Leberkrankheit; zwei Geschwister starben in früher Jugend an unbekannter Krankheit.

Die Pat. ist ledig, war vom 15. bis 50. Lebensjahre dauernd regelmäßig und beschwerdefrei menstruiert.

Fr üh ere Krankheiten: Über Kinderkrankheiten weiß Pat. keine Angaben zu machen. Mit 20 und 30 Jahren machte sie je eine Lungenentzündung durch. Sonst war sie angeblich nie ernstlich krank, nur von Jugend auf immer etwas schwächlich.

Jetzige Erkrankung: Seit 5 Jahren leidet Pat. an stetig zunehmender Engigkeit und an Herzklopfen. Seit 1 Jahre sollen sich wiederholt kurzdauernde Anfälle von Bewußtlosigkeit eingestellt haben, besonders nach psychischen Erregungen. Im AnschluB an solche Anfälle, aber auch sonst hie und $d a$ spontan auftretend, habe sie im linken Arm und Bein Zuckungen und Lühmungserscheinungen verspürt, meist für die Dauer von 2 Minuten. Anfang November 1907 zog sich Pat. eine Erkältungskrankheit zu, die mit Schnupfen, Husten, und Fieber einherging. Seitdem habe die Atemnot zugenommen und der Leib sei stärker aufgeschwollen.

Jetzige Beschwerden: Ohrensausen, Hitzegefühl im Kopf, Engigkeit auf der Brust, Herzklopfen, Husten mit Auswurf; Appetitlosigkeit, Durstgefühl, Schlaflosigkeit, Stuhlverstopfung und Brennen beim Wasserlassen.

Status pracsens (vom 22. November 1907): Kleine, grazil gebaute Frau mit schwacher Muskulatur und mäßigem Fettpolster.

Die H a u t ist blaß, die Lippen sind zyanotisch. Die Temperatur ist normal, beträgt $36,1^{\circ}$. Keine Ödeme, kein Exanthem. Halslymphdrüsen etwas geschwellt, nicht schmerzhaft.

K o p f : Pupillen reagieren. Zunge, Pharynx und Tonsillen o. B.

H a ls ohne Struma.

Th or ax lang und flach, schmal und starr, die Wirbelsäule etwas kyphotisch.

Die A t m ung ist mühsam, angestrengt; Nasenflügelatmen besteht nicht.

$\mathrm{Hu}$ s te n mittelreichlich.

Die Lungen: Grenzen : r. vorne unten 7. Rippe, 1, vorne unten 5. Rippe, beiderseits verschieblich. H. beiderseits am 11. Proc. spinosus, nicht verschieblich. Der Perkussions $\mathrm{sehall} \mathrm{im} \mathrm{allgemeinen} \mathrm{laut} \mathrm{und} \mathrm{voll,} \mathrm{mit} \mathrm{tympanitischem} \mathrm{Beiklang.} \mathrm{L.} \mathrm{H.} \mathrm{u.} \mathrm{ist} \mathrm{der} \mathrm{Schall}$ etwas höher, kaum kürzer; im r. 1. Interkostalraum desgleichen, etwas höher, nicht kürzer. Beiderseits oberhalb der Spina scapulae ist der Schall kürzer als unterhalb.

Das A temgeräu sch ist leise vesikulär; über beiden Lungen diffuses Giemen und fein- bis mittelblasiges Rasseln hörbar; 1. H. u. besonders reichlich und teilweise von klingendem Charakter; am reichlichsten $1 . \mathrm{v}, \mathrm{o}$.

Das Herz: Der Spitzensto B diffus bis zum 7. Interkostalraum noch in der Axilla zu fühlen. Auch im Epigastrium Pulsationen.

A bs olute Däm $\mathbf{p}$ fung nicht verbreitert, nicht überlagert.

H e r z m ß e : 4,7 : 8,5:17 cm; die Herzfigur erscheint längsgestellt und tiefstehend.

$\mathrm{T} \ddot{\mathrm{n}} \mathrm{e}$ : Wegen des Stöhnens der Pat. ist die Auskultation nur unvollkommen möglich, deutliche Geräusche sind nicht wahrzunehmen.

B l u tdruck: $185 \mathrm{~cm} \mathrm{H}_{2} \mathrm{O}$. P u ls etwa 160. Die peripherischen Arterien sind geschlängelt und etwas rigide.

Das A b d o m e n gleichmäßig vorgewölbt, gibt tympanitischen Schall. Bei Beklopfen des Epigastriums Plätschergeräusche. L e b e r und $\mathrm{M} \mathrm{il} \mathrm{z}$ sind nicht palpabel, jedoch ist im r. Hypochondrium eine oberflächliche Resistenz zu fühlen.

Von seiten des Nervensystems keine Besonderheiten.

Im U rin Spuren von Albumen.

Dieser Befund deutete analog den subjektiven Beschwerden auf bestehende Herzinsuffizienz hin. 
Die allgemeine Prostration, die Zyanose, der beschleunigte Puls, die dyspnoische Atmung, der Husten - als Staungsbronchitis aufgefaßt, die Leberschwellung - Resistenz unter dem r. Rippenbogen, die Albuminurie - als Zeichen der Staungsnieren, - alles das kann zur Erhärtung der Diagnose angeführt werden.

Ferner erwies sich aus dem Lungenbefunde das Bestehen eines Emphysems.

Die Analyse der Befunde am Herzen ergibt eigentlich nur wenige Anhaltspunkte für ein bestimmtes Krankheitsbild. Zwar erhellt aus einem $\mathrm{r}$. Medianabstand von 4,7 $\mathrm{cm}$ bei der kleinen, schwächlichen Frau eine Vergrößerung des.r. Vorhofes, aber bei dem Fehlen sonstiger Symptome daraus weitere Schlüsse zu ziehen, war schlechterdings unmöglich.

Unter diesen Umständen lautete die klinische Diagnose: Ins ufficientia cordis ; Emphysema; Bronchitis; Nephritis? - wozu sich im weiteren Verlaufe noch Zeichen von $\mathrm{Bronch}$ op ne u m on i e gesellten.

Die Pat. war 8 Tage in klinischer Behandlung, vom 22. bis 30. November 1907. In dieser Zeit wurden am 23. und vom 26 . bis 29 . November febrile bzw. subfebrile Temperaturen beobachtet, meist nur wenig über $37^{\circ}$, nur am 27 . November mit einer Spitze bis $38,4^{\circ}$.

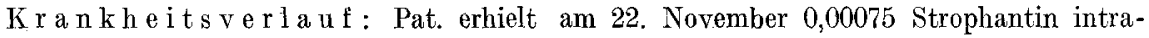
venös; ihr Befinden besserte sich nur wenig bis zum 23. November. In der Nacht auf den 24. November hin war Pat. zunächst sehr unruhig; allmählich trat Beruhigung in, Dyspnoë und Pulsfrequenz ließen nach. Am Morgen des 24. war das Sputum rubiginös, blieb so nur einige Stunden, enthielt keine besonderen Bakterien. Am Herzen waren einige Extrasystolen wahrzunehmen. Der Puls schwankte zwischen 88 und 100. Am Nachmittag stieg er plötzlich auf 170 an. Am 25. November Temperatur bis $37,1^{\circ}$; Puls 118 , irregulär und inäqual; 0,001 Strophantin intravenös war ohne Wirkung auf ihn und den Blutdruck. Weiterhin erhielt Pat. Kampher und Koffeïn. Am 27. November kam wieder eine plötzliche Steigerung der Pulsfrequenz von 90 irregulären auf 192 reguläre Pulse mit gleichzeitig einhergehender Unruhe der Pat. zur Beobachtung. Die 'Jemperatursteigerung bis $38,4^{\circ}$ veranlaßte eine eingehende Iungenuntersuchung, die eine Schallverkürzung r. h. u. ergab, in deren Bereich reichliches klingendes Rasseln zu hören war. Nachts von $12 \frac{1}{2}$ bis $1 \frac{1}{2}$ trat wieder ein ,tachykardischer Anfall" ein, 160 Pulse; darauf blieb der I'uls um 90, war irregulär und inäqual. Im Verlauf des 28. und 29. November blieb der Status im allgemeinen gleich. Gegen Abend (29. November) wurde Pat. sehr unruhig, war viel anßer Bett, so daß die Verabreichung von Morphin erforderlich war.

Am Morgen des 30. November begann sie zu verfallen; sie wurde komatös, tief zyanotisch; der Puls frequent - 144 - und irregulär und inäqual; über den Pulmones war keine deutliche Dämpfung nachweisbar; auskultatorisch diffuses Rasseln. Gegen 11 Uhr 30 Min. a. m. erfolgte der Exitus letalis.

Der Krankheitsverlauf gab somit keinen Anlaß, an der oben wiedergegebenen klinischen Diagnose eine Änderung vorzunehmen.

Der klinische Befund fand in folgender Weise durch die Sektion seine Bestätigung bzw. Erklärung.

Ich gebe zunächst das von Herrn Dr. T'il p, I. Assistenten am pathologischen Institute, verfaßte Sektionsprotokoll wieder und liefere nachher die eingehende Beschreibung des mir von Herrn Prof. Chi ari zum genaueren Studium übergebenen Herzens.

Der K ö r p e r $155 \mathrm{~cm}$ lang, von schwachem Knochenbau, schwacher Muskulatur, mager, blaß, mit blaßvioletten Hypostasen rückwärts. Die Totenstarre vorhanden.

Das $\mathrm{H}$ a a $\mathrm{r}$ braun, mit Grau gemengt.

Der $\mathrm{Hals}$ dünn. Thor a $\mathrm{x}$ schmal: Mammae atrophisch. Abdomen leicht ausgedehnt. Kopfsektion ergab normale Verhältnisse, nur etwas Atrophie des Gehirnes.

$\mathrm{Z}$ we $\mathrm{r} \mathrm{ch}$ fell r. an der 5., l. an der 6. Rippe.

$\mathrm{Hals}$ organe blaß. 
Die L u n g e $\mathrm{n}$ partiell angewachsen; in ihren Spitzen ziemlich ausgeheilte alte Tuberkulose. In ihren Bronchien reichlicher eitriger Schleim. Ihr Parenchym substanzarm, besonders an den Rändern gedunsen.

Im He r z b e u t e $120 \mathrm{ccm}$ klares Serum.

Das H e r z mäBig erweitert; 1. Vorhof und r. Ventrikel in mittlerem Grade hypertrophisch. Die beiden Zipfel der Mitralis miteinander vollständig verschmolzen, verdickt und starr, nur dort, wo die Klappeninsertionen links aneinanderstoßen, findet sich eine Kommunikation zwischen Kammer und Vorhof, zwischen den Sehnenfäden hindurch in Form mehrerer Öffnungen. L. Ventrikel in seiner Wand anscheinend dünner. Herzfleisch ohne Besonderheiten.

Die übrigen Klappen zart bis auf eine leichte Verdickung der Trikuspidalklappe und der Insertionslinien der Aortenklappen.

Intima aortae leicht fleckig verdickt.

Ösophagus blaß.

Die Organe des Unterleibes mit Staungszeichnung versehen, derb.

In der 1. Niere ein mit hämorrhagischem Hof versehener, $\frac{1}{2} \mathrm{ccm}$ großer, weißer Infarkt. Genitale wurde nicht seziert.

Magen-Darmtrakt o. B.

Pankreas und Nebennieren normal.

Demnach lautete die pathologisch-anatomische Diagnose: Emphysema pulmonum. Bronchitis suppurativa. Stenosis abnormis ostii venosi sinistri ex endoc a rditide. Hypertrophia excentrica ventriculi dextri. Dilatatio activa atrii sin. Hyperaemia mechanica universalis. Infarctus anaemicus renis sinistri. Tuberculosis obsoleta apicum pulmonum.

Meine im nachfolgenden gegebene detaillierte Beschreibung des Herzens hält sich an das in Formalin-Alkohol gehärtete und im Museum des hiesigen pathologischen Institutes unter der Nr. 5068 aufbewahrte Präparat.

Die Maße, die ich der Anschaulichkeit halber beifüge, dürfen deswegen als nur annähernd zutreffend hingenommen werden.

Falsche Vorstellungen können daraus wohl nicht erwachsen, da die eingetretene Schrumpfung in den fast normalen Teilen des Herzens von der Norm nur wenig abweichende Maßverhältnisse bedingt hat und deswegen an den pathologisch veränderten auch nur geringgradig genannt werden darf.

Im Sektionsprotokoll findet sich die Angabe, daß das - frische - Herz nur mäßig erweitert sei. Damit stimmt recht gut die Maßbestimmung ${ }^{1}$ ) im klinischen Bericht überein; daraus war; wie gesagt, auf eine Dilatation des r. Vorhofes zu schließen.

Daß der Status eine Vergrößerung der absoluten Dämpfung nach $r$. vermissen läßt, die wir als Zeichen einer Volumenszunahme des rechten Ventrikels kennen, dürfte sich unschwer aus dem Vorhandensein eines Emphysems erklären lassen, indem die gedunsenen Lungenränder das Herz von der vorderen Thoraxwand entsprechend zurückzudrängen imstande waren.

Bei der Betrachtung des Herzens im allgemeinen erscheint die Dilatation beider Vorhöfe und die Wandverdickung des 1 . ganz besonders als recht augenfällig jedoch nicht so exzessiv, wie man es bei der hochgradigen Stenose des - an letzter Stelle zu beschreibenden - 1. venösen Ostiums. hätte erwarten können.

Die Wanddicke des I. Vorhofes beträgt im Mittel $3 \mathrm{~mm}$; sie verjüngt sich gegen die Veneneinmündungsstelle hin in ziemlich beträchtlichem Grade, während sie sonst im ganzen ziemlich gleichmäßig bleibt.

1) Die Maße sind nach der Mo r it z sehen Methode bestimmt und bedeuten: r. und L. Medianabstand zur Herzlänge zwischen r. Vorhofvenenwinkel und Herzspitze. 
An der Verdickung hat nicht nur die Muskulatur, sondern auch in erheblichem Mabe das Endokardblatt seinen Anteil.

Die Innenfläche des Vorhofes ist wie mit Zucker übergossen. Die normalerweise deutlich durchschimmernde Muskulatur ist nicht sichtbar. Das Endokard ist nicht glatt, sondern zeigt zahlreiche strahlig verlaufende Leisten und Fältelungen.

Der Vorhof als Ganzes weist reichliche buchtige Ausweitungen auf. Ziemlich unberührt ist das linke Herzohr; es hat die normale Weite. Zwischen den nicht verdickten Kammuskeln haften einzelne lockere Gerinnsel. Sein Endokardüberzug ist nicht lädiert.

Der $r$. Vorhof weicht nur wenig von der Norm ab. Seine Dilatation ist schon oben erwähnt. Das Foramen ovale ist geschlossen; die Fossa ovalis scheint wegen der in die allgemeine Verdickung der linken Vorhofswand einbezogenen Verdickung der Vorhofsscheidewand nieht durch. Die Trikuspidalklappe ist nur sehr wenig verändert. Von einem anatomischen Klappenfehler kann nicht die Rede sein, es besteht eine geringe Verdickung der freien Zipfelränder, während der sonstige Klappenapparat normal zart erscheint.

Der r. Ventrikel ist etwas erweitert, seine Wand in mittlerem Grade hypertrophiert, und zwar bis zu einer Dicke von $8 \mathrm{~mm}$. Sein Endokard erscheint kaum hauchartig getrübt. Die Trabeculae carneae sind ganz dünne, nirgends besonders mächtig entwickelt oder abgeplattet. Auch die Papillarmuskeln sind vollkommen frei, von normaler Länge und Dicke; die Chordae tendineze sehr zart und nicht miteinander verwachsen.

Die Klappenzipfel erscheinen anch von der Ventrikelseite her nicht weiter verändert.

Der 1. Ventrikel wird im Sektionsprotokoll als klein bezeichnet, seine Wand dünn. In der Tat erscheint sein Lumen eng und die Wand wenigstens nicht verdickt, und im Vergleich zum r. Ventrikel tritt der Gegensatz zur Größe bei einem normalen Herzen stark hervor. Immerhin sind die Maße nicht verringert, nicht unter der Norm: die Wanddicke beträgt im Mittel $10 \mathrm{~mm}$. Zur Feststellung einer Atrophie möchte ich mich deshalb nicht entschließen. Auf diesen Punkt näher einzugehen, werde ich weiter unten Gelegenheit haben.

Das ganze Endokảrd ist diffus hauchartig getrübt, so daß das Muskelbraun nur angedeutet hindurchschimmert. Eine intensivere Trübung besteht an der Papillarmuskulatur wie auch stellenweise an den Trabeculae, welche namentlich an der Spitze und Kammerscheidewand eine deutliche Abplattung erfahren haben.

Im Vordergrunde der Veränderungen am 1. Ventrikel stehen die, welche ein Blick auf den Klappenapparat der Mitralis gleich als hochgradig zur Erkenntnis bringt. Da ist nichts von den sonst zarten Formen übriggeblieben: massiv, in breitem Relief streben die starren, verdickten Papillarmuskelgruppen zu den Segeln hin (s. Textfig. 1).

Die mediale Muskelgruppe mißt in der Mitte zwischen Ursprung und Ende in ihrer ganzen Breite $14 \mathrm{~mm}$, das zum vorderen Segel hinziehende Bündel $8 \mathrm{~mm}$, das zum hinteren $6 \mathrm{~mm}$. Zwischen beiden spannen sich schmälere und breitere fibröse Brüeken hin; außerdem sind die einander zugekehrten Flächen breit miteinander verwachsen. Ähnlich, nur noch wuchtiger bietet sich die laterale Gruppe dar. Sie miBt in ihrer Breite $20 \mathrm{~mm}$, wovon auf das vordere Bündel allein $15 \mathrm{~mm}$ kommen; das hintere ist nicht meßbar, da es hinter dem vorderen verschwindet.

Die Chordae tendineae sind, mit einzelnen Ausnahmen, die weiter unten Erwähnung finden, weitgehend verdickt, verkürzt und untereinander verwachsen, so daß sie reliefartig ausmodelliert auf der weißen Fläche des vorderen Klappenzipfels erscheinen, mit dem wenigstens die Chordae 2. und 3. Ordnung gleichfalls verwachsen sind.

Dadurch schon allein erfahren die Segel eine Verdickung. Außerdem macht sich noch eine Flächenzunahme geltend, und zwar eine Propagation gegen die Spitzen der Papillarmuskeln bis zu diesen hin, dadurch, daß die Chordae 1 . Ordnung untereinander verwachsen sind; so da $B$ sich die Segel fast ohne Abgrenzung wellig auf die Papillarmuskeln fortpflanzen.

Die mittlere Dicke der Chordae tendineae beträgt $2 \mathrm{~mm}$ gegen $0,3 \mathrm{~mm}$ im normalen Zustande. 
Wenn anch die Papillarmuskeln etwas kurz und gedrungen erscheinen, so hat doch keine hochgradige Retraktion stattgefunden. Von der Herzspitze bis zum Ende der Chordae tendineae des vorderen Klappenzipfels messe ich $65 \mathrm{~mm}$. Daß das Ganze trotzdem jenen kurzen, gedrungenen Eindruck macht, dürfte hauptsächlich auf die mächtige flächenhafte Entwieklung der Cuspis anterior zurückzuführen sein.

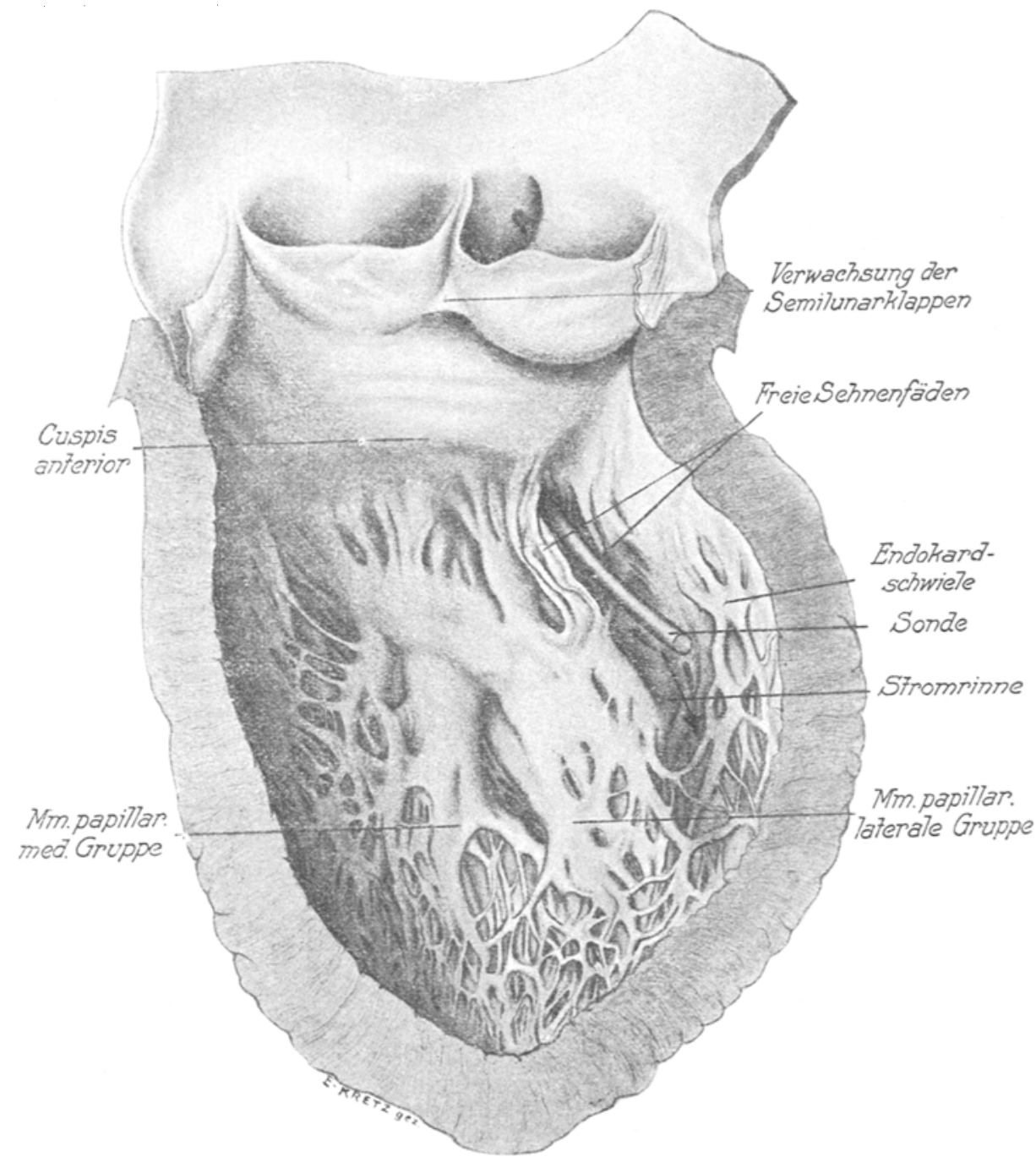

Fig. 1.

Natürlich hat das"vordere Segel vollkommen seine normale Gestalt verloren, wie in der Hauptsache schon aus der oben gegebenen Darstellung der Chordae tendineae erhellt. Seine (Ventrikel-) Fläche ist quergewellt und -gestreift, nicht aber mit Längsfalten versehen. Es zeigt keinerlei sklerotische Veränderungen, sondern ist ganz diffus verdickt. Ich erwähne das Fehlen derartiger Erscheinungen, weil man sklerotische scil. atheromatöse Prozesse am vorderen Mlappenzipfel 
neben gleichzeitig bestehenden gleichartigen Veränderungen an der Intima aortae beobachtet hat und sie als ein Vordringen derselben innerhalb einer aus der Aortenwand stammenden Schicht des vorderen Mitralklappenzipfels aufgefaßt wissen will ( $\left(\mathrm{e}\right.$ r $\left.\operatorname{ann} \mathbf{n}^{6}{ }^{1}\right)$ ).

Die Aortenwand selbst ist nur wenig fleckig verdickt; dagegen besteht an den Semilunarklappen im Aortenostium zwischen dem mittleren und lateralen Taschensegel eine kleine brückenförmige Verwachsung, und zwar zwischen ihren Insertionsstellen (s. Textabb. 1). Daraus eine Stenosierung des Ostiums anzunehmen, würde m.E. zu weit gehen, und intra vitam sind daraus wohl keine Symptome entstanden.

Wie schon oben erwähnt, sind nicht alle Chordae tendineae in gleicher Weise mit in die Verwachsung einbezogen worden.

Es sind nämlich in der l. Herzkante die am weitesten lateral gelegenen Sehnenfäden fast ganz unverändert geblieben. Sie sind nur in geringem Maße verdickt, vom Anfang bis zu ihrem Ende nicht durch Verwachsungen fixiert, so daß sie imstande gewesen sein müssen, frei im Blutstrom zu flottieren.

Es sind dies - von der Ventrikelseite aus gesehen - nur drei Fäden. Durch die zwei Zwischenräume zwischen ihnen hindurch kann man mit einer Sonde - Federkiel - in den Vorhof gelangen. Es ist das die einzige Kommunikation zwischen Vorhof und Ventrikel. Der Weg zwischen den beiden Papillarmuskelgruppen ist durch deren vollständige Verklebung verlegt. Dementsprechend verhalten sich auch die beiden. Klappenzipfel. lhre Flächen sind von medial her weithin miteinander verbacken und nur da, wo sie lateralwärts in ihren Insertionslinien aneinanderstoßen, öffnet sich die noch zwischen ihnen bestehende ziemlich seichte Kluft eben zwischen jenen Sehnenfäden hindurch in den Ventrikel.

Um nun die Stellung der Segel, $d . h$. die Lage, in der sie infolge der Verklebung fixiert sind, anschaulicher zu gestalten, greife ich zurück auf die experimentellen Untersuchungen von $\mathrm{E}$. M a g n u s - Alsleben über den Klappenmechanismus. Die von mir zu besehreibende Stellung stimmt nämlich weitgehend mit der von M a g $\mathrm{n}$ u s-Alsleben als für die Systole als normal erwiesenen überein. M a g $\mathrm{n}$ u s - Alsleben machte seine Versuche an frisch entnommenen Tierherzen, die er mit einer isoviskösen ( $10 \%$ ) Gelatinelösung füllte und dann durch Einbringen in Formalin zu einer systolischen Kontraktion reizte und damit zugleich dem Härtungsprozeß unterzog.

An den so gehärteten Herzen fand er eine Klappenstellung, die er folgendermaßen beschreibt: „Das kleine - d. h. hintere - Segel, an die Mittellinie herangeführt, steht etwas quer zur Längsachse. Das große Segel hat eine nur kleine Schwenkung gemacht, so daß es in seinem unteren Teile schräg, in seinem oberen dagegen fast parallel zur Septumwand liegt." Durch diese Knickung des unteren Teiles des vorderen Segels kommt es zu einer relativ nur geringen Berührung beider Segel, denn nur dem umgeknickten unteren Teile legt sieh ein entsprechend großer des hinteren Zipfels an. Außerdem legen sich noch die Randstreifen beider Zipfel aneinander, so daß ein plattgedrückter, mehr der hinteren Wand genäherter Trichter entsteht.

So verhält es sich auch in meinem Falle, nur daB die als Berührungsflächen gekennzeichneten Teile dauernd verwachsen sind mit Ausnahme des lateralen Randstreifens.

Einer Eigentümlichkeit der Ventrikelform muß ich noch Erwähnung tun, bevor ich mich an die Beschreibung der Klappe, wie sie sich von der Vorhofseite darstellt, begeben kann.

Es ist das eine, wie mir scheint, gut charakterisierte und sicher zu bewertende Rinne in der lateralen Ventrikelwand oder besser der lateralen Herzkante, durch die die Breitenentwicklung des Ventrikels eine Vergrößerung erfährt und in einem gewissen Gegensatz zu der Tiefe des Ventrikels steht, die recht seicht erscheint.

1) De wit z k y - Virch. Arch. Bd. 199/2 - weist nach, daß derartige - hier also fehlende Veränderungen auch an den übrigen Klappen vorkommen, und bezeichnet sie als „Ringsklerose". (Über den Bau und die Entstehung versehiedener Formen der chronischen Veränderungen an den Herzklappen; I.-Diss. Moskau.) 
Diese Rinne beginnt an der Kommunikationsstelle zwischen Vorhof und Ventrikel und hält die Richtung ein, die der Blutstrom durch das enge Ostium genommen zu haben seheint.

Diese Rinne nimmt ungefähr den kleinen Finger in sich auf, und ich möchte annehmen, daB sie dem durch das - exzentrisch gelegene - enge Ostium hindurchgepreBten Blutstrom ihre Entstehung verdankt. Sie läuft dann deutlich erkennbar zur Herzspitze hin aus. Daß an ihrem oberen, also dem Vorhof nahegelegenen Ende der Blutstrom mit ziemlich bedeutender Energie sich gegen die Ventrikelwand ergossen hat, scheint mir aus dem Umstande einleuchtend, daß sich an jener Stelle, gerade dem am meisten entwickelten Teile des Ostiums gegenüberliegend, eine ziemlich derb entwickelte Endokardschwiele befindet (s. Textabb. 1). Von hier aus dürfte der Blutstrom durch die entgegenstehende Ventrikelwand seine Ablenkung eben im Sinne jener „Strom"rinne erfahren haben.

Auf diese Verhältnisse bin ich - einen Teil der „,Bedeutung der abnormen Stenose“" vorwegnehmend - deshalb schon hier eingegangen, weil mir die einfache Registrierung einer „Stromrinne" zu wenig voraussetzungslos erschien. .

Auf solche Stromrinnen hat in sehr eingehender Weise Oestreich hingewiesen. Er fand sie an Herzen mit Mitralstenose ziemlich konstant, und zwar ganz besonders deutlich an der Septumwand, also aufsteigend von der Herzspitze zur Aortenöffnung. Auf das Vorhandensein dieses Schenkels der Stromrinne legt er deshalb besonderen Wert, weil er sie als Wirkung eines hypertrophischen Ventrikels auffassen möchte und darin einen Gegenbeweis gegen die atrophierende Tendenz des 1 . Ventrikels bei Mitralstenose erblickt (siehe auch unten).

Ich will hier noch bemerken, daß in meinem Falle der aufsteigende Schenkel fehlt. Den absteigenden bringt $O \mathrm{estreich}$ mit der Lage des verengerten Ostiums zusammen. Unter Lage versteht er nur die Richtung, wohin die Ebene, in der sich das Ostium befindet, schaut, also wohin sich der Blutstrom ergießt. Wie in den einzelnen Fällen das Ostium zum Zentrum des 1. Atrioventrikularringes lag, gibt er nicht an.

Er sagt nur, daB die Lage - bzw. besser, in seinem Sinne - die Richtung „erheblichen Schwankungen unterworfen ist und von der Art der Verwachsung und der Verkürzung abhängt".

„Im allgemeinen ist die Öffnung gegen eine mehr der Spitze als der Basis des Herzens nahe gelegene Stelle des linken Randes gerichtet" - im Gegensatze zu meinem Falle, wo diese Stelle dicht unter dem Klappenansatze liegt, also nahe der Basis. Dies gerade ist eine unmittelbare Folge der abnormen Stenose, der exzentrischen "Lage", im wahren Sinne.

Es erübrigt noch, die Klappenveränderungen zu schildern, wie sie vom Vorhof aus zu sehen sind. Beide Segel erscheinen gegen den Vorhof hin vorgewölbt, das vordere wegen seiner größeren Flächenausdehnung mehr als das hintere. Die Kuppenbreite des vorderen beträgt $24 \mathrm{~mm}$, die des hinteren $5 \mathrm{~mm}$.

Der größte Durchmesser des Annulus fibrosus ist $35 \mathrm{~mm}$; die - allein meBbare - Dicke des hinteren Segels beträgt in der Nähe der Verwachsungsstelle beider Segel $7 \mathrm{~mm}$.

Die Kuppen der Segel stoßen in einem gegen die Aortenwand hin konkav geöffneten halbmondförmigen Spalt mit unregelmäßig geformten Rändern zusammen (s. Textfig. 2). Dieser Spalt zieht sich entsprechend dem Ansatze beider Segel am Annulus fibrosus von etwas medial hinten - in der angegebenen Form - nach lateral vorn; medialwärts teilt er sich deutlich in zwei Arme, die das mediale Zwisehen- oder Hilfssegel zwischen sich fassen. Der Spalt selbst, der sich wie eine Kluft zwischen die beiden Segel einschiebt, ist in seiner medialen Partie seicht, wird nach lateral hin zunehmend tiefer - in der Mitte beträgt die 'Tiefe etwa $12 \mathrm{~mm}$. Weiter seitlich öffnet sich der Spalt in der schon oben beschriebenen Form in den Ventrikel.

Die Kluft zwischen den Segeln läßt sich am besten mit einer von sphärischen Dreiecken begrenzten Pyramide vergleichen.

Wie erwähnt ist der Rand des Spaltes rauh und unregelmäBig gestaltet, an einer Stelle durch kleine Fasern überbrückt. Frische endokarditische Exkreszenzen sind nicht vorhanden. Dagegen 
befinden sich einzelne verkalkte Herde, ein hufeisenförmiger im kleinen, hinteren”Segẹl, ein unregel. mäßig umgrenzter in dem medialen Hilfssegel.

Ein eigenartiges Verhalten zeigen die Chordae tendineae und die Teileder Papillarmuskeln, die zum hinteren Segel ziehen. Sie sind nämlich fast ganz von Veränderungen verschont geblieben, sind zart und nicht verwachsen. Dies erklärt sich wohl daraus, daß sie hinter den verklebten vorderen Papillarmuskelgruppen versteckt und fern vom Ventrikellumen lagen.

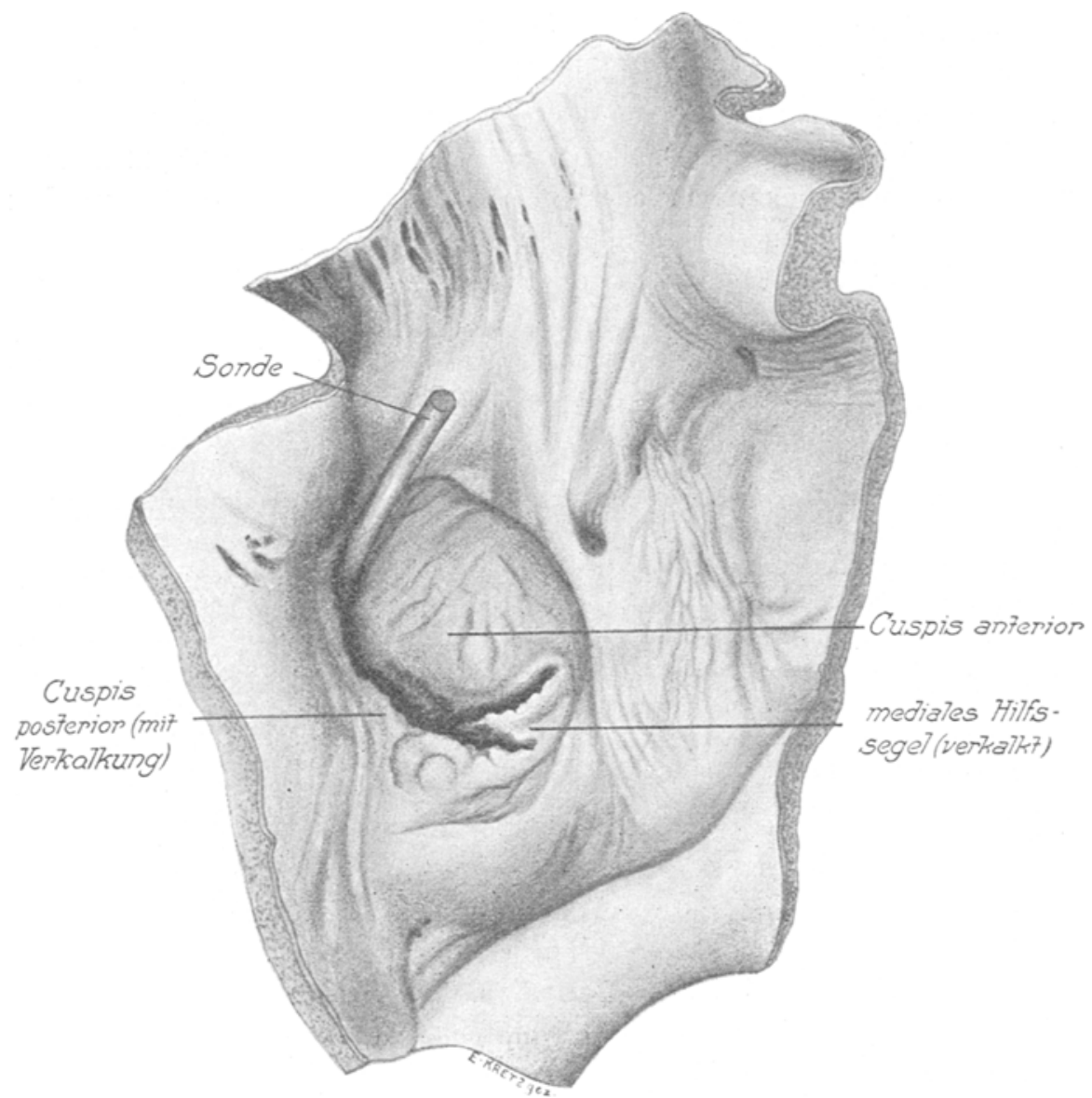

Fig. 2.

Kurz zusammenfassend möchte ich über meinen Fall sagen, daß es sich um eine hochgradige Verwachsung im Klappenapparate der Mitralis handelt, die zu einer völligen Aufhebung der normalen Kommunikationsverhältnisse geführt hat, so daß sich das Blut einen neuen Weg zum Ventrikel bahnen muBte und denselben exzentrisch nahm.

Klappenfehler der Mitralis, die auf organischen Veränderungen an den Klappen selbst beruhen, kennen wir fast nur als die Folgezustände endokarditischer Prozesse. 
Auszuschließen ist hier mit genügender Sicherheit jenes von $\mathrm{D} \mathrm{u} \mathrm{r} \mathrm{o} \mathrm{z} \mathrm{i} \mathrm{e} \mathrm{z}$ zuerst beschriebene, von ihm und zahlreichen andern, namentlich französischen, Autoren genauer definierte Krankheitsbild des „Rétrécissement mitral pur", auf das sich etwa später eine akute Endokarditis aufgepflanzt hätte, die dann die vorliegende Art der Stenose bedingt haben würde.

Bei jener auch als D u ro zi e z sche Krankheit bekannten Affektion sollen ätiologisch intrauterin wirksame Momente eine Rolle spielen.

Individuen mit der D.schen Krankheit fallen schon äußerlich auf dureh ihren schwächlichen Habitus; und gerade deswegen gehe ich auf dieses Krankheitsbild ein, weil es sich im vorliegenden Falle um eine von Jugend auf schwächliche Person handelte. Auf nähere klinische oder genetische Angaben einzugehen, würde zu weit führen. Sie finden sich ausführlich und übersichtlich zusammengestellt in der Dissertation von L. D u m ol a r d. Dagegen möchte ich kurz auf die pathologisch-anatomische Erscheinungsform, die von den französischen Autoren wohl charakterisiert ist, näher eingehen. Dabei ergeben sich die differentiell diagnostisch gegen die Annahme einer D u r o z i e z schen Krankheit in meinem Falle sprechenden Momente von selbst.

D u r o z i e ${ }^{5}$ selbst faßt das anatomisch Charakteristische in dem Satze zusammen: „Infundibulum dû à la réunion intime de deux valves restées lisses et polies."

Ein klares Bild liefert Dissit on de Larambergue: ,Es besteht kein äußeres und inneres (vorderes und hinteres, Verf.) Segel mehr: beide sind verschmolzen und bilden eine Art Hohlkegel." — ,Vom Ventrikel aus gesehen, erscheint das Ostium wie auf dem Gipfel eines abgestumpften Kegels gelegen; dabei haben die Papillarmuskeln und Sehnenfäden zueinander dieselben Beziehungen wie beim normalen Herzen, sind wohl etwas retrahiert und sklerosiert, ohne aber die Veränderungen zu zeigen, wie sie im Anschluß an infektiöse Endokarditiden entstehen." (Übersetzt aus D is sit o n.)

In meinem Falle weist nun alles darauf hin, daß es sich um eine infektiöse Endokarditis gehandelt hat.

In der Anamnese der Patientin finden sich von Infektionskrankheiten mit Sicherheit nur 2 Pneumonien und 1 fieberhafte Erkältungskrankheit 1 Monat ante mortem. Letztere kommt höchstens als Ursache für einen Nachschub in Betracht, da schon 5 Jahre lang Herzsymptome bestanden haben dürften, nach der letzten Krankheit eine Wendung zum Schlechteren im Befinden eintrat.

Pneumonien als Ursachen von Endokarditis sind wohl bekannt, aber anscheinend selten. Strümpell sagt, daß sich zuweilen bei Sektionen von Pneumonien eine geringe frische Endokarditis findet; er mißt ihr aber keine klinische Bedeutung zu.

Im allgemeinen ist man viel eher geneigt, die Endokarditis als Begleiterscheinung rheumatischer Affektionen, speziell des akuten Gelenkrheumatismus, aufzufassen.

Dazu geben die Statistiken eine gewisse Berechtigung; zwar schwanken die Zahlen in ziemlich beträchtlichen Grenzen. So gibt S e hulz e bei einer Ätio- 
logie von $76,57 \%$ Infektionskrankheiten überhaupt die Arthritis rheurn. acuta mit $34,52 \%$ an; G. L e u c h berechnet auf $81,34 \%$ sogar $65,6 \%$, und $\mathrm{S}$ t r ü m pell gibt $52,76 \%$ an (zitiert nach C. ' $\mathrm{T} \mathrm{h}$ o r e l).

Wie sich andere ähnliche Affektionen wie Anginen usw. im Verhältnis dazu stellen, ist nicht $z u$ bestimmen. Ob ich im vorliegenden Falle einen Gelenkrheumatismus oder eine nicht beachtete andere Infektionskrankheit als Ursache annehmen soll, wage ich nicht zu entscheiden.

Die Endokarditis, die ich als Grund des Klappenfehlers annehme, ist allem Anscheine nach symptomlos verlaufen. Bis sich dann als Folge der Endokarditis klinisch ein Klappenfehler manifestierte, konnten mehrere Monate bis zu einem Jahre vergehen, und ehe dann subjektive Beschwerden auftreten mußten, Jahre und Jahrzehnte ( $\mathrm{B}$ a $\mathrm{m}$ b e r g e r).

Fünf Jahre ante mortem haben in meinem Falle die Beschwerden begonnen. Damals wird also schon ein Klappenfehler bestanden haben. In welcher Form? War es damals schon eine Stenose, oder überwog eine Insuffizienz?

Nach den Untersuchungen von $\mathrm{Z}$ a h $\mathrm{n}$, deren Resultate von H. Rit ter mit Erfolg differentialdiagnostisch verwertet wurden, hinterläßt eine Insuffizienz der Valvula mitralis im Endokard des Vorhofes zwischen Ostium und der Einmündungsstelle der linken Lungenvenen wellenförmige Verdickungen.

Derartige Veränderungen konnte man in meinem Falle nicht registrieren, so daß ich mich für berechtigt halte, anzunehmen, daß die Stenose gleich zu Anfang in den Vordergrund getreten ist.

Wie ist ein solcher Hergang zu denken?

Nach Vir chow kann man bei gewissen Individuen von einer besonderen Disposition zu Klappenerkrankungen reden. So glaubt Virch ow der Chlorose und den damit zusammenhängenden Anomalien im Gefäßapparate eine Rolle zuschreiben zu müssen.

Ferner gibt es an den verschiedenen Klappen Prädilektionsstellen zur Ansiedlung von Keimen bzw. Entzündungsvorgängen, und zwar sind es in erster Linie die Teile, die einer stärkeren Zerrung ausgesetzt sind. V i r c h o w sagt: „Unter den Klappenteilen der Mitralis ist es vorwiegend der vordere Zipfel, der durch seine Befestigung an der Aortenmündung die größte Zerrung zu erleiden hat." Es herrscht nun die Vorstellung, daß an besonders gezerrten Zipfelteilen etwa nach einer Endothelläsion infolge der Zerrung sich entweder Bakterien ansiedeln oder direkt Thromben bilden. Nächst dem vorderen Segel wären es - nach Virchow - die Chordae tendineae, besonders da, wo ihre Teilung in dünnere und dickere Fäden stattfindet, die für jene Prozesse am meisten in Betracht kommen.

Nach Zi e gl er, R o m berg, Jürgensen u. a. entstehen die endokarditischen Produkte als entzündliche Reaktion auf z. B. durch Bakterien gesetzte Gewebsnekrosen. Es kommt zu thrombotischen Auflagerungen, die, wenn sie klein sind, gewöhnlich ganz durch Bindegewebe ersetzt werden; sind sie groß, so bleibt oft ein größerer Teil von ihnen erhalten, der später der Schrumpfung und Verhalkung anheimfällt. Dadurch erfahren die Klappenteile eine Verdickung und häufig auch Schrumpfung. Die entzündeten Klappenteile, die häufig miteinander in Berührung kommen und infolge schon eingetretener Starrheit länger in Berührung bleiben als normal, kommen zur Verklebung, zur festen Verwachsung miteinander, sei es nun, daß davon die Zipfel oder die Sehnenfäden und Papillarmuskeln betroffen werden. 
Eine Stenose im speziellen entsteht nach $\mathrm{R}$ i b b e r t dann, wenn sich die Zipfelklappen infolge der beschriebenen Veränderungen in „querer Richtung verkürzen“. _- „Die Stenose ist um so stärker, je mehr sich die Entzündung von dem freien Rande bis zur Basis einerseits ausdehnt und je mehr andererseits auch die Verwachsung der Sehnenfäden die Klappenfläche gleichsam nach unten verlängert und dann an der zirkulären Schrumpfung teilnehmen läßt."

Diese Verhältnisse dürften für meinen Fall recht zutreffend sein, mit dem einen Unterschiede, daß in jenen Fällen eine konzentrische Verengerung das Resultat ist, was in meinem Falle nicht eingetreten ist.

Jenes muß aber als Regel betrachtet werden. Einer andern Vorstellung begegnet man nicht. So sagt z. B. N i e m e y e r: In den bedeutendsten Graden von Stenose sind die Ränder der beiden Klappenzipfel von ihrer Basis aus so miteinander verwachsen, daß nur der mittlere Teil unvereinigt bleibt und daß die Mitralklappe einen Trichter bildet. Fast gleichlautend äußert sich J ü rg e n s e n.

Daß nun abweichend davon in meinem Falle sich der Prozeß mehr einseitig abgespielt hat, erklärt sich vielleicht so, daß es zunächst medial am Ostiumrande aus irgendeinem Grunde zur Bildung einer größeren thrombotischen Auflagerung $\mathrm{kam}$. Als seine Reste figurieren die Kalkeinlagerungen im hinteren Zipfel und medialen Hilfssegel. Dadurch, daß der Thrombus einen Teil des Ostiums verlegte und gewissermaßen die beiden Segel an der medialen Berührungsstelle mit einer Brücke verband, legte er deren Bewegung in diesen Teilen lahm, bedingte so eine längerdauernde innigere Berührung und weiterhin die Verklebung. Gleichzeitig lokalisierte sich die Endokarditis an den Papillarmuskeln und Sehnenfäden. Da aber der Blutstrom schon frühzeitig wegen der einseitigen Segelverwachsung mehr nach lateral geleitet wurde, konnte sich hier wegen der kräftigeren Bewegungen der Teile keine Verwachsung ausbilden, und es kam nur zu einer geringen Verdickung und mit der alimählichen oder sprungweise erfolgenden Progression der Erkrankung zu der beschriebenen abnormen Form der Stenose.

Unter welcher Form die fragliche Endokarditis einhergegangen ist, läßt sich nicht mit Sicherheit entscheiden. Im Grunde genommen ist es auch unwesentlich, ob aus der ursprünglich akuten Endokarditis eine chroniseh fibröse Form wurde, oder ob sich der Verlauf auf eine rekurrierende Endokarditis hinausspielte.

Ähnliche Fälle sind mir in der Literatur nicht begegnet, sei es, daB sie zu den Seltenheiten gehören, sei es, daß sie nicht aufgefallen sind.

Die meisten Autoren begnügen sich damit, die Ostien als vermindert durchgängig, spalt- oder trichterförmig zu beschreiben.

Oestreich, s. o., legt Wert auf die Stellung des Ostiums in Beziehung zur Vorhofventrikelachse, über die Lage zum Annulus fibrosus gibt er nichts an.

Dagegen finde ich bei $\mathrm{Baumbach}$ eine Angabe über verschiedene Stenosenformen, von denen mir eine als Vorstufe meiner Form gelten kann.

B. rubriziert u. a. 13 reine Stenosen und findet sie schlitz-, spalt-, loch-, trichter-, halbmond- und $\mathrm{kn}$ opflo $\mathrm{ch} \mathrm{f}$ or mig. 
In meinem Falle erscheint der Spalt des Ostiums halbmondförmig und knopflochähnlich zugleich, nur daß von dem „Knopfloch" nur der rundliche Teil noch mit dem Ventrikel in direkter Verbindung steht.

Die Frage nach der Bedeutung, die der Stenose beizumessen ist, hat $\mathfrak{u}$. a. auch einige Punkte zu berücksichtigen, die bis in die letzten Jahre Gegenstand lebhafter Kontroversen waren.

Relativ einfach ist die Bewertung der Wirkung auf Kreislauf und Herz im allgemeinen.

Wie schon oben bemerkt, möchte ich die Klappenveränderung als reine Stenose auffassen. Man könnte dem entgegenhalten, daß die starren Klappen nicht vermocht hätten, die stenosierten Stellen im Ostium ausreichend zu verschließen, da in der Tat das Postulat, das man zur AusschlieBung einer Insuffizienz gestellt hat: bewegliche freie Ränder, in meinem Fall nicht erfüllt ist.

Ich kann mir aber sehr wohl vorstellen, daß eben die freigebliebenen Sehnenfäden sich wie zu einer Spindel zusammenlegten und so die Öffnung ausfüllten.

Von den Folgen, die eine Mitralstenose im allgemeinen für die Konfiguration des Herzens in sich birgt, waren vorhanden die Dilatation und Hypertrophie des linken Vorhofs, die Dilatation und Hypertrophie des rechten Ventrikels, die Dilatation des rechten Vorhofes. Die Frage des linken Ventrikels soll weiter unten erörtert werden. Stauungserscheinungen boten die Lungen und die Unterleibsorgane. Der Infarkt in der linken Niere erklärt sich aus dem Vorhandensein von thrombotischen Prozessen im linken Herzen. Die Herzinsuffizienz, die ja im Vordergrund der klinischen Erscheinungen stand, kann zum Teil auf mangelhafte Ernährung des Herzmuskels infolge der geringen Füllung des arteriellen Systems, zum Teil auch auf den nach den Untersuchungen von v. Krehl mit jedem Herzklappenfehler einhergehenden myokarditischen Schädigungen beruhen, die der letzte Grund einer jeden Dekompensation bedeutet.

Von den Veränderungen am linken Ventrikel, die der Mitralstenose zuzuschreiben sind, haben das Vorhandensein der Stromrinne und der Endokardschwiele und sonstige Einzelheiten bereits oben genügende Erwähnung gefunden.

Nicht leicht zu entscheiden ist die Frage: In was für einem Zustande befindet sich der linke Ventrikel?

Vergleiche ich seine Maße mit den $\mathrm{Buhlschen} \mathrm{Mittelzahlen,} \mathrm{so} \mathrm{komme}$ ich, wenn ich die Schrumpfung berücksichtige, doch wohl an eine dem Individuum entsprechende mittlere Normalzahl heran.

Eine Zahl von $11,85 \mathrm{~mm}$ als mittlere Dicke von $\mathrm{B}$ a u $\mathrm{mbachs}$ Herzen, die, wenn sie das Mittel der mittleren Dicken darstellt, nach Vier ordts Kritik, den Anforderungen entspricht, dürfte in meinem Fall wohl als erreicht angesehen werden.

Allerdings habe ich nirgends Angaben darüber gefunden, wie sich die Maße in verschiedenen Herzphasen verhalten und in welchen Phasen die Autoren die 
Herzen zu Gesicht bekamen, die zum Zweck der Klärung der Ventrikelfrage Messungen anstellten. Bei der Leiche findet man die Herzen schlaff oder kontrahiert, und so wäre es denkbar, daß beim Vergleich von Zahlenwerten sich Mißverständnisse ergeben könnten.

Die sicherste Art der Bestimmung, die M ü 1 I e r sche Methode der Wägung der einzelnen Herzabschnitte, ist meines Wissens nur von $\mathrm{H}$ i r s $\mathrm{h}$ angewandt worden. Vielleicht beruhen die vielen einander widersprechenden Resultate der verschiedenen Untersucher zum Teil auf diesen Äuß̧erlichkeiten. Andrerseits sollten sie zur Genüge dartun, daß sich eine Norm über das Verhalten des linken Ventrikels nicht aufstellen läßt. $R$ itter weist deshalb derartige Versuche in dem 1. Schlußsatz seiner Dissertation zurück.

Wie viele z. B., auch die älteren Autoren vor ihm, wie $B$ a $\mathbf{m}$ b e r g e r, Friedreich, v. Dusch, Niemeyer, Rosenstein, dann Strümpell, Gerhardt u. a. m., glaubt auch Ritter einen hypertrophischen linken Ventrikel bei Mitralstenose auf irgendwelche Komplikationen zurückführen zu müssen, sei es, daß sie im Herzen selbst oder im Kreislauf irgendwo liegen.

Es würde zu weit gehen, auf alle diese Angaben einzugehen. Sie haben Gegnern der Lehre von der ,atrophierenden“ Tendenz des linken Ventrikels, die einen hypertrophischen Ventrikel als Ausdruck kompensatorischer Tätigkeit als die Norm ansehen möchten -- wie Lenhartz, Baumbach, Oest re i c h-, nicht genügt. Aber auch die Ansichten dieser Autoren sind nicht unwidersprochen geblieben, so daß, wie die Dinge heute stehen, mit beiden bzw. drei Möglichkeiten gerechnet werden muß: Atrophie, normale Wand, Hypertrophie.

Eine Atrophie wird man dann erwarten dürfen, wenn im Herzmuskel reichlich degenerative Vorgänge neben der Mitralstenose einhergehen und der Muskel selbst in einer Komplikation nicht einen Ansporn zur Steigerung seiner Kraft erhält. Normal b]eibt ein Muskel, wenn die myokarditischen Veränderurgen entweder fehlen oder doch nur gering sind -- oder wenn sie durch gleichmäßige Hypertrophie kompensiert werden; Hypertrophie endlich kann zur Ausbildung kommen bei Vorhandensein von Komplikationen zu einer Zeit, wo der Muskel kräftig zu reagieren imstande war.

Für diejenigen Fälle, in denen Hypertrophie ohne Komplikationen besteht, ist bis jetzt keine ausreichende Erklärung gebracht.

In meinem Falle würde es sich um einen Ventrikel mindestens von normaler Wandstärke handeln.

Eine atrophierende Tendenz möchte ich deswegen nicht annehmen, d. h. alo gegeben bei Mitralstenose, da sie, wie gesagt, andern Faktoren zuzuschreiben ist, als etwa solchen, die der Mitralstenose ihr Dasein verdanken. 
Eine Tatsache aus der Krankengeschichte dürfte noch von Interesse sein, nämlich das Auftreten tachykardischer Anfälle.

Mackenzie beschreibt sie als gelegentliches Symptom bei Mitralstenose. Auch Martius will sie ,"lediglich als Symptom, d.h. Begleiterscheinung bezüglich Folge eines primären andern Krankheitsvorganges", z. B. gerade als Symptom bei Klappenfehlern. M a $r$ t i u s zieht das Primäre in einer akuten, meist anfallsweise auftretenden Herzerweiterung. M a c k e $\mathbf{n}$ z i e dagegen faßt diese als Wirkung auf. Er sieht den Grund der ,paroxysmalen Tachykardie"* bei Mitralstenose darin, daß auf Grund pathologischer Veränderungen im Herzmuskel der Ursprungsort der Herzkontraktionen zeitweise von der normalen Stelle fort an eine weiter unten gelegene verlegt ist, und zwar von den Utberresten des Sinus in die Utberreste des primitiven Herzschlauches (Atrioventrikulärknoten). Solche pathologische Veränderungen sollen in Infiltrationsherden oder in aus solchen entstandenen Narben bestehen, die ihren Sitz entweder im atrioventrikulären Bündel selbst oder in dessen Nähe haben. Die Störung wird dadurch hervorgerufen, daß infolge jener anatomischen Veränderungen die Reizleitung eine Hemmung erfährt und daß dann jene erwähnte Stelle, der Atrioventrikulärknoten nach $\mathrm{T}$ a w a $\mathrm{r}$ a, die Führung übernimmt.

Jene pathologisch-anatomischen Veränderungen fand $\mathrm{Keith}$ - zitiert nach Mackenzi e -.- in entsprechenden Fällen bei Patienten, die zum Teil an rheumatischen Affektionen gelitten hatten. Aus diesem Grunde möchte ich als möglich annehmen, dah die im vorliegenden Falle beobachtete Tachykardie gleichen Ursprungs war.

Speziell wurde aber von mir in dieser Richtung nicht untersucht, um das interessante Präparat zu schonen.

\section{Lit eratur.}

1. B a m b e r g e r, Lehrbuch der Krankh. des Herzens, 1857. -- 2. B a u $\mathrm{m} \mathrm{b}$ a c h, Über das Verhalten des linken Ventrikels bei der Mitralstenose. D. Arch. f. klin. Med. Bd. 48, 1891. 3. D is siton d e L a r a m b e $\mathrm{rg}$ u e, Essai sur le rétrécissement mitral pur. Thèse de Paris 1900. - 4. D u m o la r d, L é o $\mathrm{n}$, Contribution à l'étude de l'origine congénitale du rétrécissement mitral pur. Thèse de Lyon 1902. - 5. D u r o z i e z, Rétrécissement mitral. Union médicale 1892. - 6. H e r rm a n n. Beitrag zu den Erkrankungen der Herzklappen. Inaug.-Diss. Halle 1903. - 7. H i r s ch, Úber die Beziehungen zwischen der Körpermuskulatur und dem Herzmuskel. D. Arch. f. klin. Med. Bd. 64. -- 8. J ü r g e n s e n, Klappenfehler. Nothnagels Spezielle Path. u. Therapie Bd. 15. Wien 1903. - 9. K rehl, Beitrag zur Path. der Herzklappenfehler. D. Arch. f. klin. Med. Bd. 46. - 10. L e n hart z, tber das Verhalten des linken Ventrikels bei Mitralstenose. Münch. med. Wschr. Nr. 22, 1890. - 11. M a c k e n z ie, Krankheiten des Herzens. Lehrb. - 12. M a g n u s-Alsleben, E., Zum Mechanismus der Mitralklappe. Arch. f. experim. Path. usw. 1907, Bd. 57. - 13. M a r ti us, Allgemeine Pathologie des Kreislauiss. Lubarsch-Ostertags Ergebn. Bd. 1, 2. Abt., 1895. - 14. N i e me yer, Spezielle Pathologie und Therapie. Lehrb. - 15. Oestreich, Das Verhalten der linken Kammer bei den Erkrankungen der Valvula mitralis. Arch. f. path. Anat., Physiol. u. f. klin. Med. 1898, Bd. 151. - 16. R i b bert, Jehrb. der spez. Path. - 17. R it t e r, Das Herz bei Mitralstenose. Inaug.-Dissert. Halle 1909. - 18. Ro mberg, Endocarditis: D. Arch. f. klin. Med. Bd. 1894, 1 u. 2. - 19. s t r ü m p e 11, Lehrb. der spez. Path. u. Ther. Bd. 1. - 20. Th or e 1, C., Pathologie der Kreislauforgane. Lubasrch-Ostertag, Ergebn. Bd. 9, 1. Abt., 1903. - 21. V i e r o r d t, Daten und Tabellen. 1. Aufl. - 22. V i e r o r d t, Kritik bzw. Referat über Baumbachs Arbeit s. o. 2. Friedländers Fortschr. d. Med. Jahrg. 10, 1892. 23. $\mathrm{V}$ i r c h ow, Ges. Abhdln. - 24. $\mathrm{Z}$ a h n, Uber einige anatomische Kennzeichen der Herzklappeninsuffizienzen. Verhdl. des XIII. Kongr. f. inn. Med. 1895. - 25. Z i e g l e r, Lehrb. d. spez. Path. 